\title{
Locoregional options in the management of cholangiocarcinoma: single center experience
}

\author{
Hussein Baydoun ${ }^{1}$, Harley Meirovich ${ }^{1}$, Gilbert Maroun ${ }^{1}$, Natalie Coburn², Elizabeth David ${ }^{1}$ \\ ${ }^{1}$ Department of Vascular and Interventional Radiology, Sunnybrook Health Sciences Center, University of Toronto, Toronto, Canada; ${ }^{2}$ Department \\ of General Surgery, Sunnybrook Health Sciences Center, University of Toronto, Toronto, Canada \\ Contributions: (I) Conception and design: H Baydoun, G Maroun; (II) Administrative support: H Baydoun, E David; (III) Provision of study materials \\ or patients: All authors; (IV) Collection and assembly of data: H Baydoun, G Maroun; (V) Data analysis and interpretation: H Baydoun, G Maroun; \\ (VI) Manuscript writing: All authors; (VII) Final approval of manuscript: All authors. \\ Correspondence to: Elizabeth David, MD. Department of Vascular and Interventional Radiology, Sunnybrook Health Sciences Center, University of \\ Toronto, Toronto, Canada. Email: Elizabeth.david@sunnybrook.ca.
}

Background The purpose of this case series is to assess the safety and efficacy, as well as the overall survival (OS) and progression free survival (PFS) of patients with intrahepatic cholangiocarcinoma (ICC) treated with percutaneous ablation, transarterial arterial chemoembolization (TACE) or a combination of both at our institution.

Methods: Ten patients with pathological diagnosis of adenocarcinoma treated at out institution between January $1^{\text {st }} 2013$ and January $1^{\text {st }} 2019$ were reviewed. Three patients received a combined TACE and ablation treatment, three patients received TACE without ablation and four patients were treated with ablation only. Ablation technical success was determined by absence of residual tumor in the ablation zone on follow-up imaging one-month post-ablation. TACE response was assessed using the Modified Response Evaluation Criteria in Solid Tumors (mRECIST). Technical success was defined as injection of chemoembolic material in the involved liver lobes. PFS and OS were calculated from the date of diagnosis.

Results: In the TACE and radiofrequency ablation (RFA) group, OS was 12, 55 and 56 months; PFS was 5, 6 and 32 months, one patient died and two others remain alive. In the TACE group, OS was 29, 10 and 5 months; PFS was 15, 10 and 4 months. All three patients remain alive. In the ablation group, OS and PFS were 16, 31, 30 and 40 months. All patients remain alive. Overall, 9 of 10 patients are alive, with a Median OS and PFS of 29.5 and 15.5 months, respectively, with some patients remain alive over four years following initial presentation.

Conclusions: Our study shows that ablation and TACE in combination with more traditional modalities such as chemoradiation and surgical resection can extend survival in patients with ICC significantly. Locoregional therapy is well tolerated with only minor adverse events. The use of stereotactic body radiation therapy (SBRT) with ablation demonstrated the synergistic nature of using multiple lines of interventions.

Keywords: Overall survival (OS); radiofrequency ablation (RFA); transarterial arterial chemoembolization (TACE); intrahepatic cholangiocarcinoma (ICC); Progression free survival (PFS); locoregional treatment

Submitted Oct 09, 2019. Accepted for publication Apr 10, 2020.

doi: 10.21037/apm-19-362

View this article at: http://dx.doi.org/10.21037/apm-19-362

\section{Introduction}

The incidence of intrahepatic cholangiocarcinoma (ICC) is increasing worldwide and accounts for approximately $20 \%$ of cholangiocarcinoma/gallbladder malignancies, with an overall incidence of 0.7 cases per 100,000 adults in the United States (1). The long-term survival of patients with unresectable ICC is dismal, with 5 to 8 months survival (2) and less than $5 \%$ to $10 \%$ of patients alive 5 years after 
diagnosis (3). Predisposing factors to development of ICC include primary sclerosing cholangitis, hepatobiliary parasitosis, intrahepatic lithiasis and chronic viral hepatitis (1).

ICC is often diagnosed at an advanced stage in a symptomatic patient (4). Only $15-30 \%$ of ICC patients present with resectable disease at time of diagnosis $(2,5)$. Curative surgical resection with negative tumour margins can be achieved in less than $30 \%$ of patients with ICC, with an estimated median survival time of 36 months. Furthermore, current evidence does not support the option of liver transplantation in this patient population (6).

ICC has a metastatic predilection for the liver. Therefore, locoregional therapies may play a role in local disease control (6). However, a minority of patients are candidates for thermal ablation, as most tumors are too advanced for this treatment option (4). A systematic review by Han et al. (7) on radiofrequency ablation (RFA) in the treatment of unresectable ICC reported a technical success rate of $80 \%$ to $100 \%$, defined as complete ablation of the tumor shown on imaging follow-up 1 month after RFA. Furthermore, pooled 1-year, 3-year and 5-year survival rates were $82 \%, 47 \%$ and $24 \%$, respectively, and the median survival time ranged from 20 to 60 months.

There is limited data on the role of transarterial arterial chemoembolization (TACE) in this patient population, with limited studies showing overall survival (OS) ranging from 10 to 35 months (4). Ray et al. (8) performed a metaanalysis which included 16 studies evaluating the efficacy of TACE in ICC. The median survival from time of diagnosis was 15.7 months, which compares favorably to reported OS rates of 5 to 8 months in systemic chemotherapy. Furthermore, more than three fourths of subjects showed a response or stable disease as per RECIST criteria. The ideal chemotherapeutic protocol is also unclear. Certain authors reported on the safety and efficacy of conventional chemotherapeutic regimens such as Mitocyn C, gemcitabine and cisplatin $(9,10)$. Data on safety and efficacy of drugeluting beads loaded with Doxorubicin (DEBDOX) and drug-eluting beads loaded with Irinotecan (DEBIRI) is comparatively limited. Aliberti et al. (11) reported on the safety and efficacy of DEBDOX in 11 patients with a median survival of 13 months. Venturini et al. (12) compared the efficacy of DEBDOX and DEBIRI, concluding that DEBIRI was more effective than DEBDOX as a second-line treatment for hepatic metastases from cholangiocarcinoma. Their study was however limited by their small sample size (10 patients receiving a total of 32 TACE sessions).

The primary objective of this case series is to assess the safety and efficacy, as well as the OS and progression free survival (PFS) of patients with ICC treated with percutaneous ablation, TACE or a combination of both at our institution.

\section{Methods}

The study was approved by the institutional Research Ethics Board Committee of Sunnybrook Health Sciences Center (No.016-2019) and conducted in accordance with the Declaration of Helsinki (as revised in 2013). Because of the retrospective nature of the research, the requirement for informed consent was waived. A retrospective, singleinstitutional review was performed of all cases of TACE, RFA, microwave ablation (MWA) and percutaneous ethanol injection (PEI) performed at our institution between January 1st 2013 and January 1st 2019. Cases performed for cholangiocarcinoma were retained and confirmation of surgical pathology or biopsy results was made via patient records. A total of 10 patients, five male and five females, were reviewed (Table 1).

Collected data points included patient demographics, clinicopathological characteristics, type of intervention (surgical, medical, radiation, locoregional therapy), and OS. Pathological diagnosis of an adenocarcinoma without clinical or imaging evidence of an extrahepatic primary was presumed to represent a cholangiocarcinoma. Staging was based on the most up-to-date guidelines from the American Joint Committee on Cancer (Carcinoma of the Intrahepatic Bile Ducts). Initial RFA and MWA technical success was determined by absence of residual tumor in the ablation zone on follow-up imaging (CT scan or MRI) 1-month post-ablation. Technical success at 3 months, 6 months, 1 year and 2 years when available was also recorded. Technical failure of ablative therapy was defined as the presence of residual tumor (nodular or mass like enhancement 1-month post-procedure). TACE response was assessed using the Modified Response Evaluation Criteria in Solid Tumors (mRECIST). Technical success was defined as injection of chemoembolic material in the involved liver lobes. Periand post-procedural complications were documented. PFS and OS were calculated from the date of diagnosis.

\section{Treatment algorithm}

At our institution, hepatobiliary tumors are presented at multidisciplinary cancer conferences which are attended by hepatobiliary surgeons, medical oncologists, radiation oncologists and interventional radiologists. The 
Table 1 Demographic data

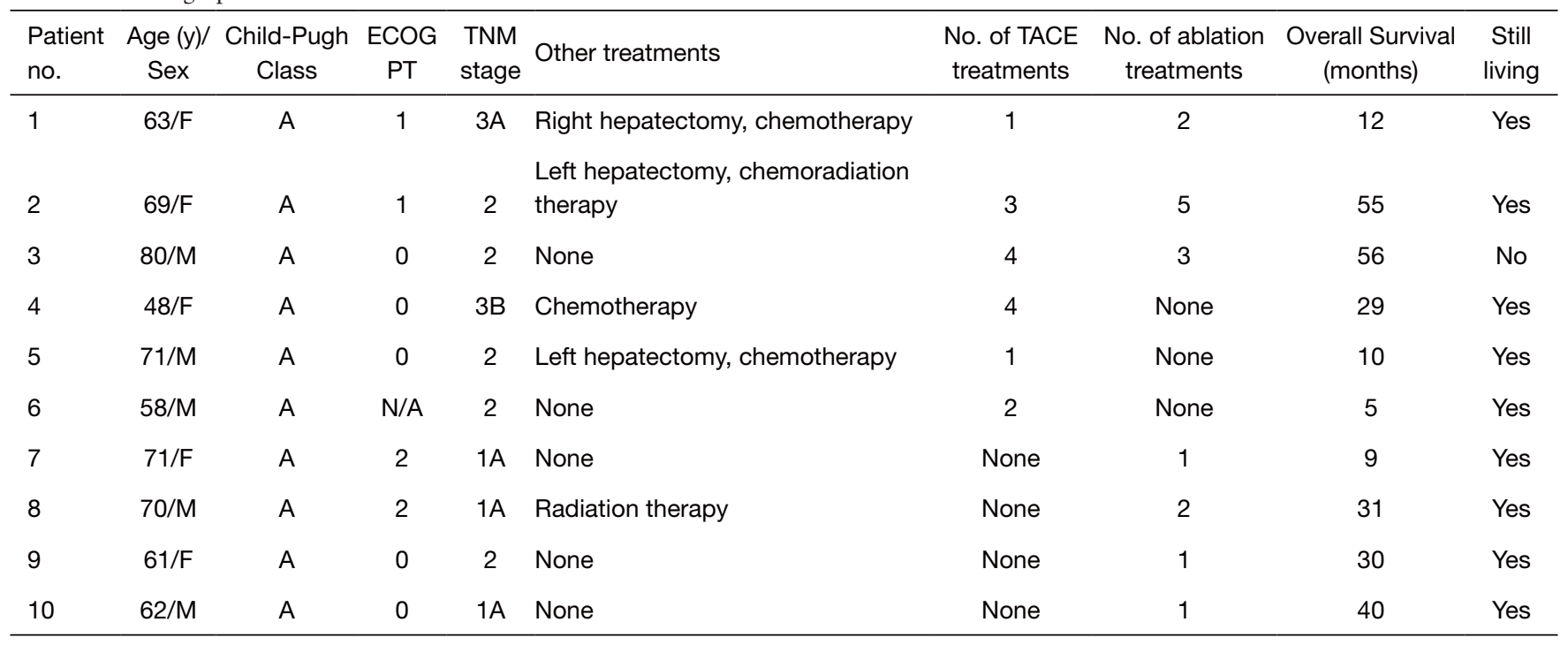

TACE, transarterial arterial chemoembolization.

treatment decision is made on a case-by-case basis in a multidisciplinary setting. Patients referred for locoregional treatment are then assessed in clinic by the interventional radiology team. Combination therapy, including combined systemic and locoregional therapy or combined locoregional therapy (ablation, radiation and TACE) are offered in select cases. The number of TACE and ablation sessions required are determined by tumor size and number of lesions.

\section{Follow-up}

Follow-up schedule is dependent on treatment outcome and need for additional therapy. Follow-up CT scan or MRI is performed 1 month following the last locoregional treatment; no imaging is needed between treatment sessions if the patient's clinical course is uneventful.

\section{Technique}

At our institution locoregional ablation is most frequently performed under combined ultrasound and CT guidance. RFAs are performed with a Medtronic Cool-tip RF ablation system E series using either a single electrode with a 2 or $3 \mathrm{~cm}$ active tip, a cluster electrode with a $2.5 \mathrm{~cm}$ active tip or a switching controller kit which is constituted of three switching needles which are positioned in a triangular distribution. Select RFAs are performed using the AngioDynamics Starburst RFA electrode. MWAs are performed with an Angiodynamics Solero MWA system using a $13 \mathrm{G}$ electrode with a $2 \mathrm{~cm}$ active tip at 60,100 or 140 watts. The decision to use either of these systems is based on tumor size, location and operator preference. We aim for an ablation zone with a $1 \mathrm{~cm}$ margin beyond the border of the mass on imaging when feasible with a minimum acceptable margin of $5 \mathrm{~mm}$. Hydrodissection is used to displace surrounding critical structures during ablation. Pre procedural antibiotics are not routinely given unless patient is high risk (post sphincterotomy or hepaticojejunostomy). Procedure is done with conscious sedation and patients are discharged the same day.

There is no currently agreed upon best practice for chemotherapeutic agent in TACE. DEBDOX and DEBIRI have both been used at our institution. The particle size is determined on a case-by-case basis, varying with operator preference and anatomic considerations. Patients routinely receive pre-procedure antibiotics, either a combination of Metronidazole and Cefazolin in patients at low risk for post-procedure abscess or Piperacillin/Tazobactam in patients at high risk for post-procedure (sphincterotomy or hepaticojejunostomy). The procedure is performed under conscious sedation and the patients are admitted overnight for monitoring, they are typically discharged the next morning.

\section{Results}

\section{Patients treated with TACE and RFA}

Three patients received a combined TACE and ablation 

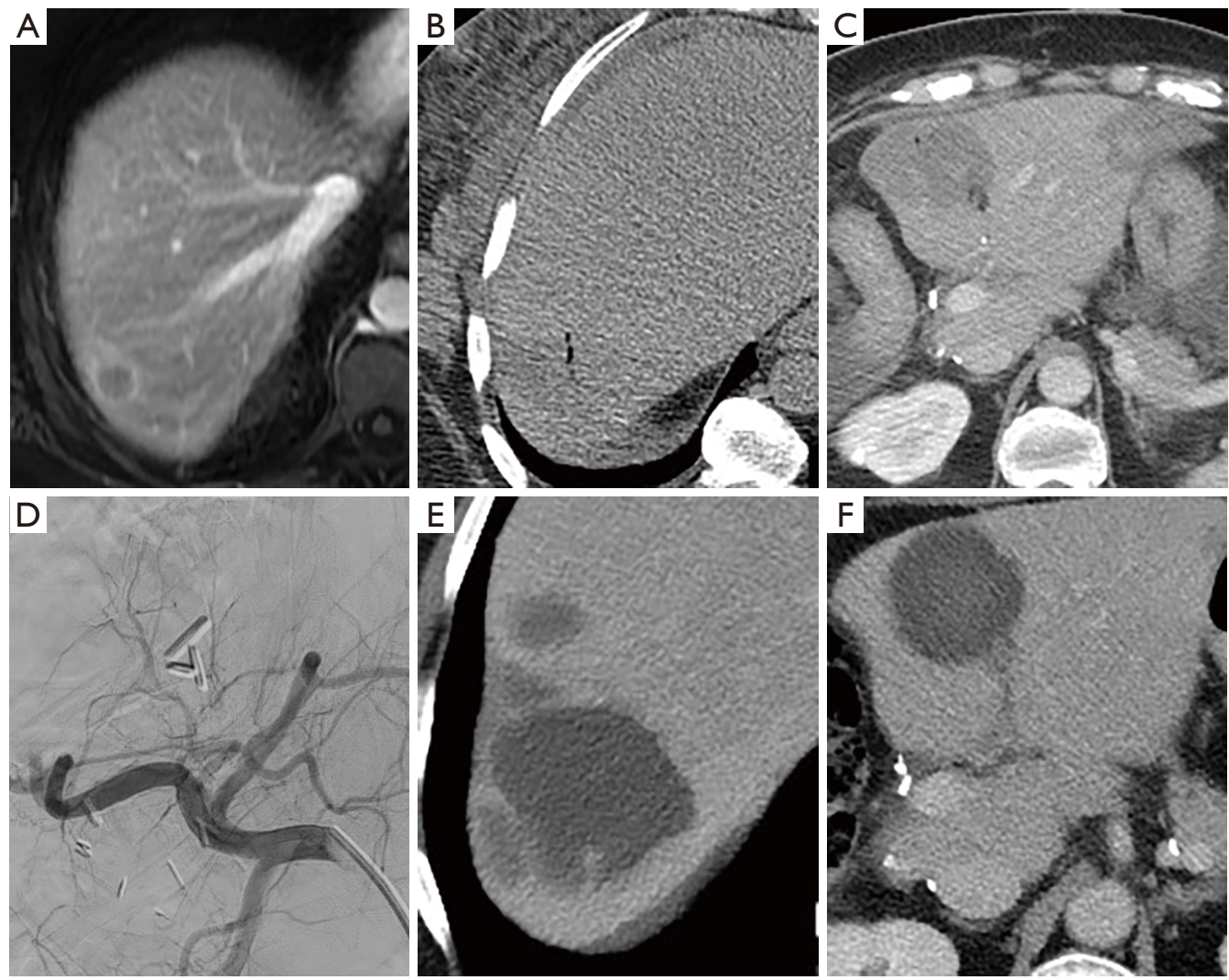

Figure 1 A 63 -year-old female with multifocal cholangiocarcinoma, disease recurrence following right hepatectomy. (A) Pre-procedure MRI revealed 4 bilobar metastases the largest in segment 7 measuring $1.2 \mathrm{~cm}$ and located near the liver dome. (B,C) Post-ablation CT scans following bilobar RFA performed in 2 sessions. (D) Additional right lobar TACE for control of residual disease, completion angiogram shows sluggish flow in the right lobar territory. (E,F) Follow-up CT scan 12 months following presentation. There is development of recurrent disease at the margins of the ablation and chemoembolization zones. The patient remains alive to this day. RFA, radiofrequency ablation; TACE, transarterial arterial chemoembolization.

locoregional treatment. Two patients initially underwent partial hepatectomy with neoadjuvant chemotherapy for one patient, and adjuvant chemoradiation for the other. Although surgical margins were free of tumor, both patients developed recurrent disease on three and four-month postoperative imaging, respectively, and were offered additional locoregional therapy. The third patient was treated initially with a combination of TACE and ablation only (Table 1).

All three patients had multifocal disease at the time of first locoregional treatment, measuring up to $4.9 \mathrm{~cm}$ in maximum diameter. A total of 10 ablations were performed, one MWA and nine RFAs. Eight ablations were technically successful. One ablation failure was attributed to lesion size, measuring $4.9 \mathrm{~cm}$ in diameter, a second ablation was performed successfully. The other ablation failure was due to the mass being located near the liver dome in segment 8 , with limited visualization (Figure 1). The residual disease was treated with stereotactic radiation successfully.

Eight TACE sessions were performed, four with DEBDOX and four with DEBIRI. All sessions were technically successful, with no post-procedural complications. Two patients had progressive disease on post-procedural imaging following their last TACE session, whereas one had partial response.

OS was 12, 55 and 56 months; PFS was 5, 6 and 32 months. The third patient, with an OS of 56 months (Figure 2), died whereas the other two remain alive. ECOG status and Child-Pugh scores remained stable throughout their treatments. 

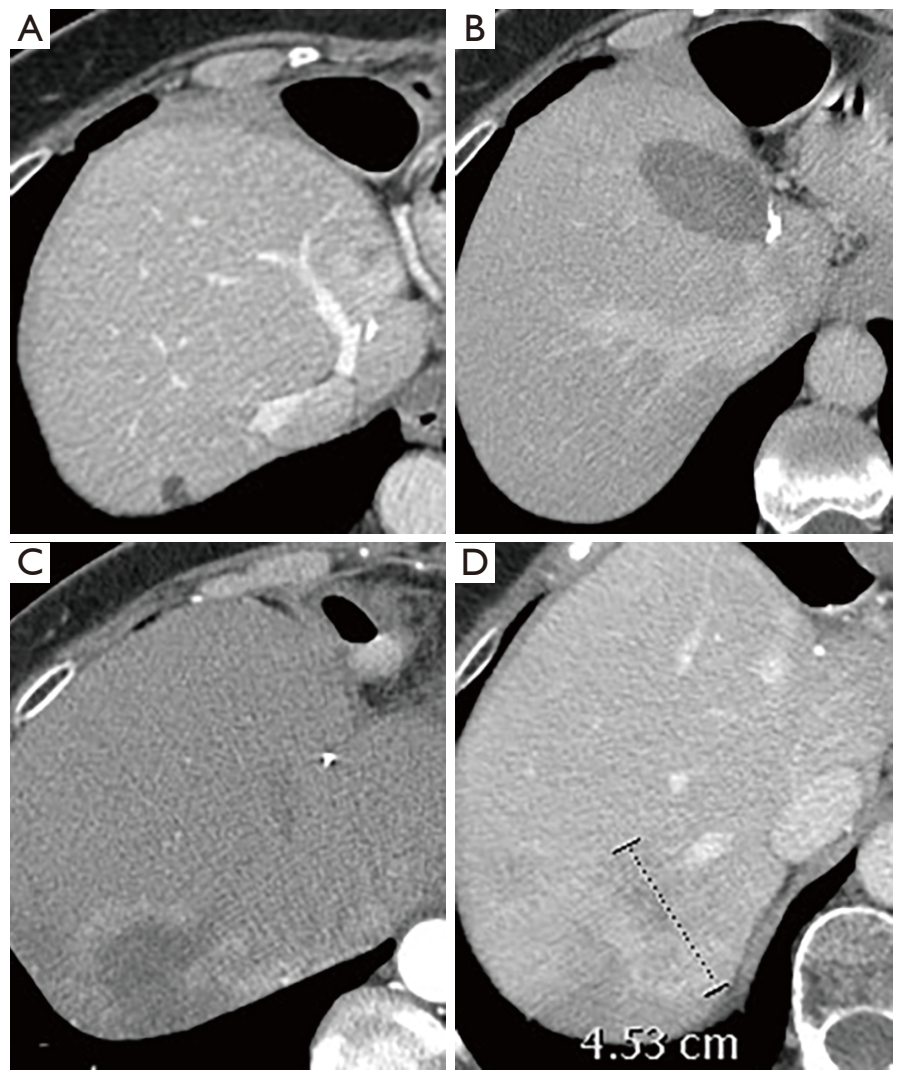

Figure 2 A 69-year-old female with multifocal cholangiocarcinoma initially treated with left hepatectomy and SBRT of her bilobar metastases. (A) $0.7 \mathrm{~cm}$ segment $4 \mathrm{~A}$ recurrence at the resection margin which was not amenable to SBRT given its proximity to the stomach. Note the segment 7/8 hypodensity from prior SBRT. (B) Post-RFA CT scan shows adequate ablation zone without residual or injury to surrounding structures. (C) Following 18 months of stability, her right lobar disease progressed with the largest mass measuring $4.5 \mathrm{~cm}$ in segment 7. Segment 4A ablation zone was stable (not shown). (D) CT scan following combination of several ablations and TACE treatments, the patient has developed recurrent disease at the ablation margins. She remains alive 55 months following initial diagnosis. SBRT, stereotactic body radiation therapy, RFA, radiofrequency ablation.

\section{Patients treated with TACE}

Three patients received TACE without ablation. One patient was initially treated with cisplatin/gemcitabine followed by gemcitabine/capecitabine; this was then followed by four TACE sessions. No patients in this cohort were eligible for surgical resection. One patient had both gastric cancer and a left lobar cholangiocarcinoma, for which they initially underwent TACE and neoadjuvant FOLFOX followed by a left hepatectomy after downsizing of the tumor following interventions. Of the two other patients, one received a combination of cisplatin/gemcitabine followed by gemcitabine/capecitabine and TACE. The third patient was treated with TACE only (Table 1).

All three patients had multifocal disease at the time of locoregional treatment, measuring up to $14 \mathrm{~cm}$ in maximum diameter. A total of seven TACE were performed, three with DEBDOX and four with DEBIRI. All sessions were technically successful, with no post-procedural complications. All three patients had partial response on imaging one month after their last TACE session. There were no complications.

OS was 29, 10 and 5 months; PFS was 15, 10 and 4 months. All three patients remain alive. ECOG status and Child-Pugh scores remained stable throughout their treatments.

\section{Patients treated with ablation}

Four patients were treated with ablation only (Table 1 ). 

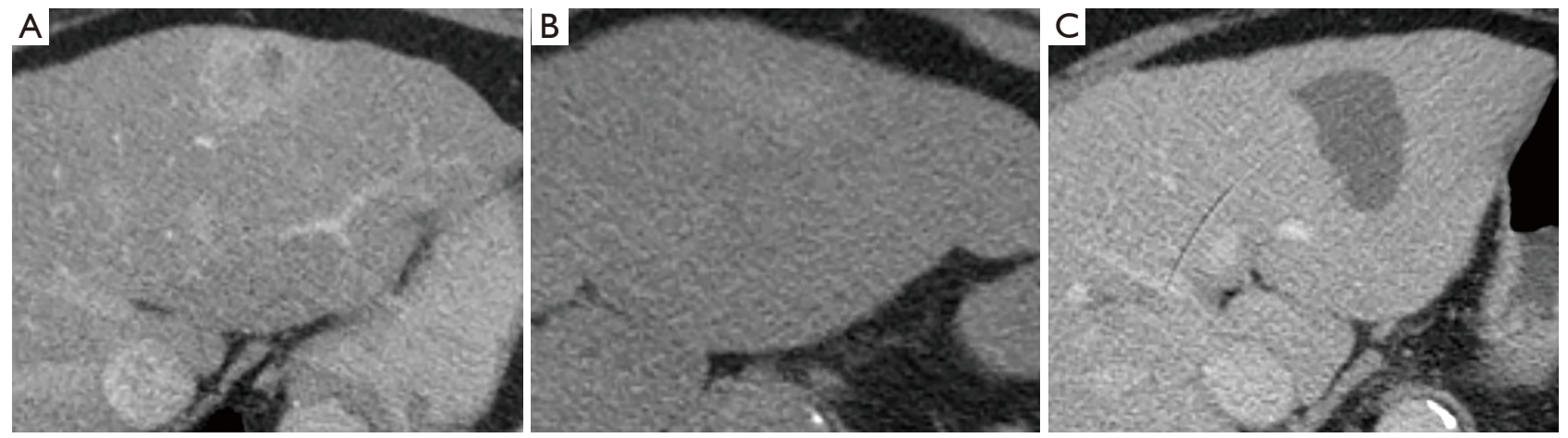

Figure 3 A 72-year-old man with focal ICC and underlying cirrhosis. Unresectable due to the severity of the liver disease. Not a candidate for transplant. (A) Pre-ablation contrast-enhanced CT scan; $4 \mathrm{~cm}$ segment 2 mass without washout (arterial phase not shown) in a patient with background liver cirrhosis. (B) Post RFA non-enhanced CT scan; adequate ablation zone without immediate complications. Spontaneous hyperdensity represents hemorrhage within the ablation zone, an expected finding. Contrast-enhanced CT scan 1-month post RFA showed no residual enhancing tissue (not shown) (C) Contrast-enhanced CT 1-year post ablation; Ablation zone with coagulative necrosis, no residual enhancement to suggest recurrent tumor. ICC, intrahepatic cholangiocarcinoma; RFA, radiofrequency ablation.

None of these patients received chemotherapy or underwent a surgical resection. Three patients presented with a solitary mass, the largest measuring $4.7 \mathrm{~cm}$ at time of ablation. The fourth patient presented with two masses, measuring up to $1.5 \mathrm{~cm}$ at time of ablation.

Five RFA sessions were performed. One patient had a 4 $\mathrm{cm}$ mass which was successfully treated in one session using an Angiodynamics StarBurst XL system (Figure 3). Another patient had a $4.4 \mathrm{~cm}$ mass which required two ablation sessions using a Medtronic Cool-tip RFA single electrode approach due to limited visualization, this was followed by stereotactic body radiation therapy (SBRT) due to the presence of residual tumor. Both other patients were treated in one session using a Medtronic Cool-tip RFA single electrode, with technically satisfactory results.

OS and PFS were 16, 31, 30 and 40 months. All patients remain alive today. ECOG status and Child-Pugh scores remained stable throughout their treatments.

\section{Discussion}

ICC is uncommon and patients frequently present with advanced disease, which limits treatment options. Only 30\% of patients are surgical candidates at time of presentation. Furthermore, most patients die from complications of locally advanced disease such as biliary obstruction and cholangitis as opposed to distant metastases. For these reasons, it is important to better understand the role of non-surgical locoregional therapies.
Out of seven patients treated with RFA, technical success was confirmed on follow-up imaging in all ablations performed in patients with one or more masses measuring $4 \mathrm{~cm}$ or less in maximum diameter. Ablation failures were in patients with masses measuring 4.7 and $4.9 \mathrm{~cm}$ in two cases (Figure 4), with an additional failure in a $1.2 \mathrm{~cm}$ mass which was located near the liver dome in segment 8 . Lesion size is an important predictor of technical success following radiofrequency ablation, with the rate of residual disease increasing in masses larger than $3 \mathrm{~cm}$ in maximum diameter. Proximity to large vessels can also limit RFA effectiveness due to a "heat sink" effect, however this can be mitigated with the use of MWA. Our failed segment 8 ablation was secondary to limited lesion visualization. Out of 15 ablation sessions in seven patients, two patients developed minor adverse events which were managed conservatively, with no major adverse events. This is consistent with the overall low risk of major complications following liver ablation in the literature. Out of five patients with technically successful RFA, four had no local recurrence at least 1-year post-treatment.

Patients undergoing TACE had a higher tumor burden and more advanced disease staging than patients treated with RFA alone. Patients undergoing TACE were also more likely to undergo surgical resection and chemoradiation. Out of six patients treated with TACE, four showed partial response and two showed progressive disease on 1-month follow-up imaging. Of the patients who had partial response, three showed disease progression on 3 -month follow-up. This highlights the difficulty with 

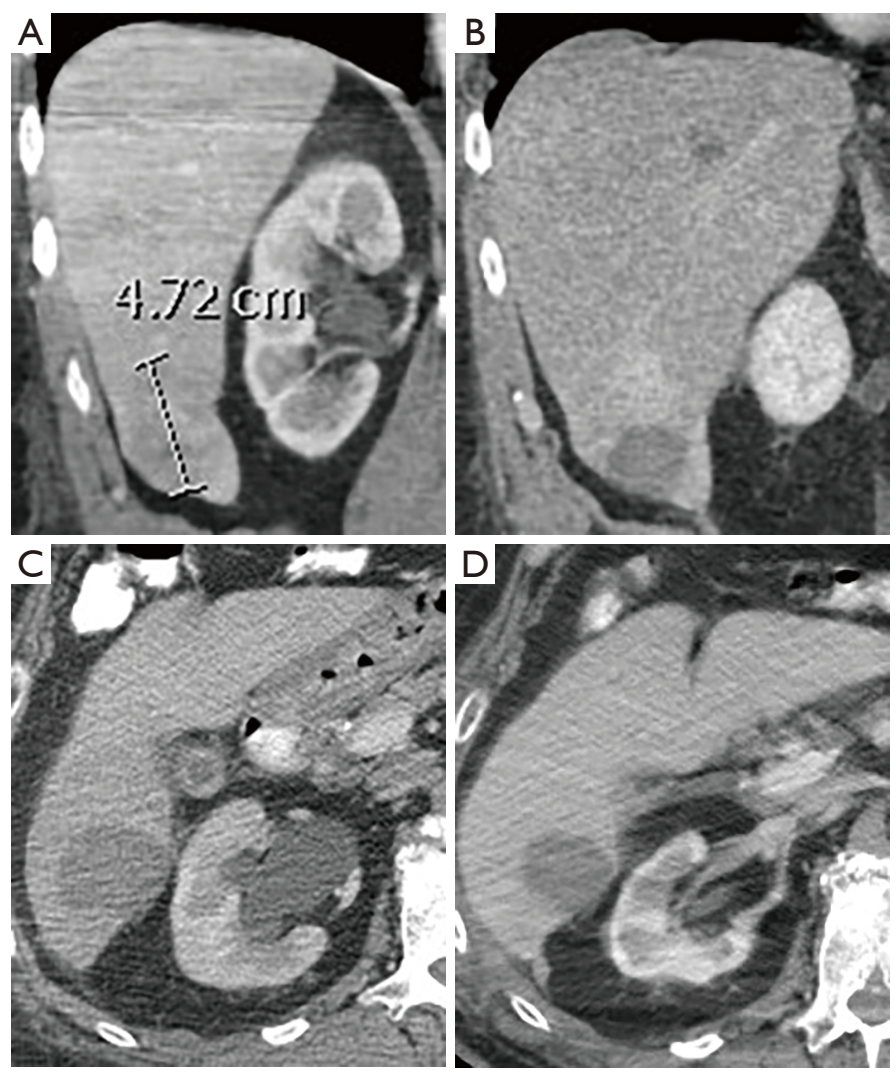

Figure 4 A 70-year-old man with segment 6 cholangiocarcinoma. (A) $4.7 \mathrm{~cm}$ segment 6 mass, no background liver cirrhosis. (B) CT scan following RFA, there is residual tumor enhancement at the superior and posterior (not shown) aspect of the ablation zone. (C) CT scan following second RFA session, adequate ablation zone without residual tumor enhancement. (D) Follow-up CT scan performed 16 months following the second RFA session, there is no recurrent disease. The patient remains alive to this day. RFA, radiofrequency ablation.

local disease control in patients who have advanced disease. The procedure was however well tolerated, with no major complications.

Median OS and PFS were 29.5 and 15.5 months, respectively, with some patients who remain alive over four years following initial presentation. Although certainly limited by our sample size, these results surpass the reported survival in the literature and are certainly promising.

Our study suffers from its retrospective nature and the small size of our cohort. It is also a heterogeneous study group, with varying treatment modalities. There is heterogeneity in chemoembolic agent used, ablation type and treatment combination. Due to paucity of data on best practice in this patient population, it is unclear what chemoembolic agent is best. Furthermore, we have limited follow-up data on three of our patients who presented within the last year from time of writing.

\section{Conclusions}

Our study shows that ablation, and TACE in combination with more traditional modalities such as chemoradiation and surgical resection can extend survival in patients with ICC significantly. Locoregional therapy is well tolerated with only minor adverse events. Our RFA results highlight the importance of proper patient selection, with both technical failures encountered in patients with tumors larger than $4 \mathrm{~cm}$ in diameter. However, the use of SBRT with ablation demonstrated the synergistic nature of using multiple lines of interventions. Our study reinforces the findings of previous publications which also show improved outcomes and supports the use of locoregional therapies in the management of ICC. 


\section{Acknowledgments}

Funding: None.

\section{Footnote}

Data Sharing Statement: Available at http://dx.doi. org/10.21037/apm-19-362

Conflicts of Interest: All authors have completed the ICMJE uniform disclosure form (available at http://dx.doi. org/10.21037/apm-19-362). Elizabeth David serves as an unpaid editorial board member of Annals of Palliative Medicine from Jul 2019 to Jun 2021. The other authors have no conflicts of interest to declare.

Ethical Statement: The authors are accountable for all aspects of the work in ensuring that questions related to the accuracy or integrity of any part of the work are appropriately investigated and resolved. The study was approved by the institutional Research Ethics Board Committee of Sunnybrook Health Sciences Center (No. 016-2019) and conducted in accordance with the Declaration of Helsinki (as revised in 2013). Because of the retrospective nature of the research, the requirement for informed consent was waived.

Open Access Statement: This is an Open Access article distributed in accordance with the Creative Commons Attribution-NonCommercial-NoDerivs 4.0 International License (CC BY-NC-ND 4.0), which permits the noncommercial replication and distribution of the article with the strict proviso that no changes or edits are made and the original work is properly cited (including links to both the formal publication through the relevant DOI and the license). See: https://creativecommons.org/licenses/by-nc-nd/4.0/.

\section{References}

1. Amin MB, Edge S, Greene F, et al. editors. AJCC Cancer Staging Manual. 8th edition. Springer International Publishing: American Joint Commission on Cancer, 2017.

2. Burger I, Hong K, Schulick R, et al. Transcatheter Arterial Chemoembolization in Unresectable Cholangiocarcinoma: Initial Experience in a Single Institution. J Vasc Interv Radiol 2005;16:353-61.
3. Mavros MN, Economopoulos KP, Alexiou VG, et al. Treatment and Prognosis for Patients With Intrahepatic Cholangiocarcinoma: Systematic Review and Metaanalysis. JAMA Surg 2014;149:565-74.

4. Padia SA. Intrahepatic Cholangiocarcinoma. Tech Vasc Interv Radiol 2015;18:227-35.

5. Buettner S, van Vugt JL, IJzermans JN, et al. Intrahepatic cholangiocarcinoma: current perspectives. Onco Targets Ther 2017;10:1131-42.

6. Razumilava N, Gores GJ. Cholangiocarcinoma. Lancet 2014;383:2168-79.

7. Han K, Ko HK, Kim KW, et al. Radiofrequency ablation in the treatment of unresectable intrahepatic cholangiocarcinoma: systematic review and meta-analysis. J Vasc Interv Radiol 2015;26:943-8.

8. Ray CE Jr, Edwards A, Smith MT, et al. Metaanalysis of survival, complications, and imaging response following chemotherapy-based transarterial therapy in patients with unresectable intrahepatic cholangiocarcinoma. J Vasc Interv Radiol 2013;24:1218-26.

9. Vogl TJ, Naguib NN, Nour-Eldin NE, et al. Transarterial chemoembolization in the treatment of patients with unresectable cholangiocarcinoma: Results and prognostic factors governing treatment success. Int J Cancer 2012;131:733-40.

10. Park SY, Kim JH, Yoon HJ, et al. Transarterial chemoembolization versus supportive therapy in the palliative treatment of unresectable intrahepatic cholangiocarcinoma. Clin Radiol 2011;66:322-8.

11. Aliberti C, Benea G, Tilli M, et al. Chemoembolization (TACE) of unresectable intrahepatic cholangiocarcinoma with slow-release doxorubicin-eluting beads: preliminary results. Cardiovasc Intervent Radiol 2008;31:883-8.

12. Venturini M, Sallemi C, Agostini G, et al. Chemoembolization with drug eluting beads preloaded with irinotecan (DEBIRI) vs doxorubicin (DEBDOX) as a second line treatment for liver metastases from cholangiocarcinoma: a preliminary study. Br J Radiol 2016;89:20160247.

Cite this article as: Baydoun H, Meirovich H, Maroun G, Coburn N, David E. Locoregional options in the management of cholangiocarcinoma: single center experience. Ann Palliat Med 2021;10(2):1784-1791. doi: 10.21037/apm-19-362 